\title{
MODELING CYCLIC WAVES OF CIRCULATING T CELLS IN AUTOIMMUNE DIABETES
}

\author{
JOSEPH M. MAHAFFY* AND LEAH EDELSTEIN-KESHET ${ }^{\dagger}$
}

\begin{abstract}
.
Type 1 diabetes (T1D) is an autoimmune disease in which immune cells, notably T-lymphocytes target and kill the insulin-secreting pancreatic beta cells. Elevated blood sugar levels and full blown diabetes result once a large enough fraction of these beta cells have been destroyed. Recent investigation of T1D in animals, the non-obese diabetic (NOD) mice, has revealed large cyclic fluctuations in the levels of $\mathrm{T}$ cells circulating in the blood, weeks before the onset of diabetes [23], but the mechanism for these oscillations is unclear. We here describe a mathematical model for the immune response that suggests a possible explanation for the cyclic pattern of behaviour. We show that cycles similar to those observed experimentally can occur when activation of $\mathrm{T}$ cells is an increasing function of self-antigen level, whereas the production of memory cells declines with that level. Our model extends previous theoretical work on T cell dynamics in T1D [14], and leads to interesting nonlinear dynamics, including Hopf and homoclinic bifurcations in biologically reasonable regimes of parameters. The model leads to the following explanation for cycles: High rates of beta cell death, and corresponding elevation of self-antigen, shut off memory cell production, leading to a gap in the population of activated T cells. Once peptide has been cleared by nonspecific mechanisms, the memory pool is renewed, and the cyclic behaviour results.
\end{abstract}

Key words. Autoimmune diabetes; type 1 diabetes, $\mathrm{CD}^{+} \mathrm{T}$ cells, cycles, homoclinic bifurcation; mathematical model

1. Introduction. Type 1 Diabetes (T1D) is an autoimmune disease in which pancreatic beta cells are killed by the immune system, shutting off insulin secretion, and resulting in elevated blood glucose. The disease affects young people, severely impacting their health, and requiring perpetual insulin injection. Finding cures and/or treatment to replace the beta cells (e.g., by transplanting islets from organ donors) remains problematic, mainly because the damage is caused by the body's own immune system, which also attacks the transplant.

Studying autoimmune diabetes in humans presents ethical and clinical challenges. Therefore, animals with diabetic tendency, including non-obese diabetic (NOD) mice are used to gain a basic scientific understanding of the disease. In NOD mice, Type 1 diabetes arises when populations of immune cells called T cells become primed to specifically target and kill beta-cells. Such cytotoxic T cells belong to a class of lymphocytes displaying a surface marker called CD8. (Hence, denoted CD8 ${ }^{+} \mathrm{T}$ cells). We first briefly describe the background immunology, and then present the detailed aspects specific to diabetes, the data on circulating $\mathrm{T}$ cells, and our model.

1.1. Immunology Primer. For an excellent survey of immunology, see [9]. T cells mature in the thymus, where those that cross-react with self-proteins are normally eliminated to prevent autoimmunity. After this period of development, they are released, circulate, and migrate to lymph nodes. In the lymph nodes, T cells interact with antigen presenting cells (APC's) that display stimuli, consisting of a small fragment of antigen protein (i.e., a peptide of about 9 amino acids in length) held inside a cleft of a larger protein (named major histocompatibility complex, or MHC for historical reasons) [4]. The peptideMHC complex (p-MHC for short) interacts with specific receptors on the surface of the T cells ("T cell receptors", abbreviated TCR's). The strength, duration, and number of such interactions experienced by a given $\mathrm{T}$ cell determines its subsequent fate $[24,26,15,27,21]$. Within the right range of affinity to and quantity of p-MHC encountered, T cells with the appropriate specificity undergo activation, and the immune response is initiated.

Under normal conditions, antigen presenting cells display antigens that are derived from foreign proteins, such as viral or bacterial coat proteins. Then, appropriately specific $\mathrm{T}$ cells are primed to form a large battalion of effector cells to combat the infection. Activated T cells proliferate, undergoing about 6 cell divisions. Their daughters are mostly effector cells (also called cytotoxic T-lymphocytes, or CTL's), efficient and specific killers that seek out and destroy target cells. These effector cells, though deadly, are relatively short-lived [5]. A few daughters of activated $\mathrm{T}$ cells are memory cells that retain the same specificity but have no immediate effect $[8,25]$. However, when the stimulus (e.g., the same foreign antigen) is encountered for a second time, memory cells can be activated rapidly to mount a faster immune response.

In autoimmune diseases such as type 1 diabetes, the antigen peptide derives from normal proteins in the host. Infection or other injury can expose such proteins and initiate the disease, but once in progress,

\footnotetext{
* Nonlinear Dynamical Systems Group, Computational Sciences Research Center, Department of Mathematical Sciences, San Diego State University, San Diego, CA 92182

${ }^{\dagger}$ Department of Mathematics and Institute of Applied Mathematics, University of British Columbia, 1984 Mathematics Road, Vancouver, BC, Canada V6T 1Z2. 
successive killing of targeted cells, and consequent release and exposure of more self-antigen can sustain the inappropriate immune response. As the immune system is a complex web of nonlinear interactions between cells, chemicals, and tissues, rich dynamical behaviour can be expected, and indeed does occur. Our first goal in this paper is to point out interesting immunological dynamics to an audience of applied mathematicians. Our second goal is to present a plausible explanation of the cycles in autoimmune diabetes observed by [23], based on an established set of known and hypothesized interactions.

1.2. Autoimmunity in type 1 diabetes. It has been shown that normal development of NOD mice includes a wave of programmed cell death (apoptosis) of pancreatic beta cells shortly after birth $[18,19]$. In these same experiments, it was also determined that clearance of the apoptotic cells (by macrophages, nonspecific cells of the innate immune system) is reduced in NOD mice, leading to the conjecture that material from these dead beta cells forms self-antigen that triggers the autoimmune response. Previous modelling efforts have focused on such early initiation events [12, 13], but here we are mainly concerned with later stages in which the adaptive immune system is involved.

A number of proteins, including insulin, have been implicated as self-antigens in type 1 diabetes. Most recently, experimental collaborators in Calgary (in the laboratory of P. Santamaria) have identified a new dominant self-antigen: IGRP (glucose-6-phosphatase catalytic subunit-related protein), a protein of beta-cells whose normal function is yet to be determined. A fragment of this protein (consisting of amino acids 206-214) is the "peptide" to which most $\mathrm{CD}^{+} \mathrm{T}$ cells in T1D react [10]. The discovery of this specific self-antigen in NOD mice followed years of experiments in which libraries of artificially synthesized peptides were used to identify and label $\mathrm{T}$ cells $[2,1,6]$. Use of tetramer probes (constructed of four copies of peptide-MHC with a fluorescent tag) allowed careful investigation of the levels and dynamics of these cells, by enhancing the ability to label cells that were previously undetectable.

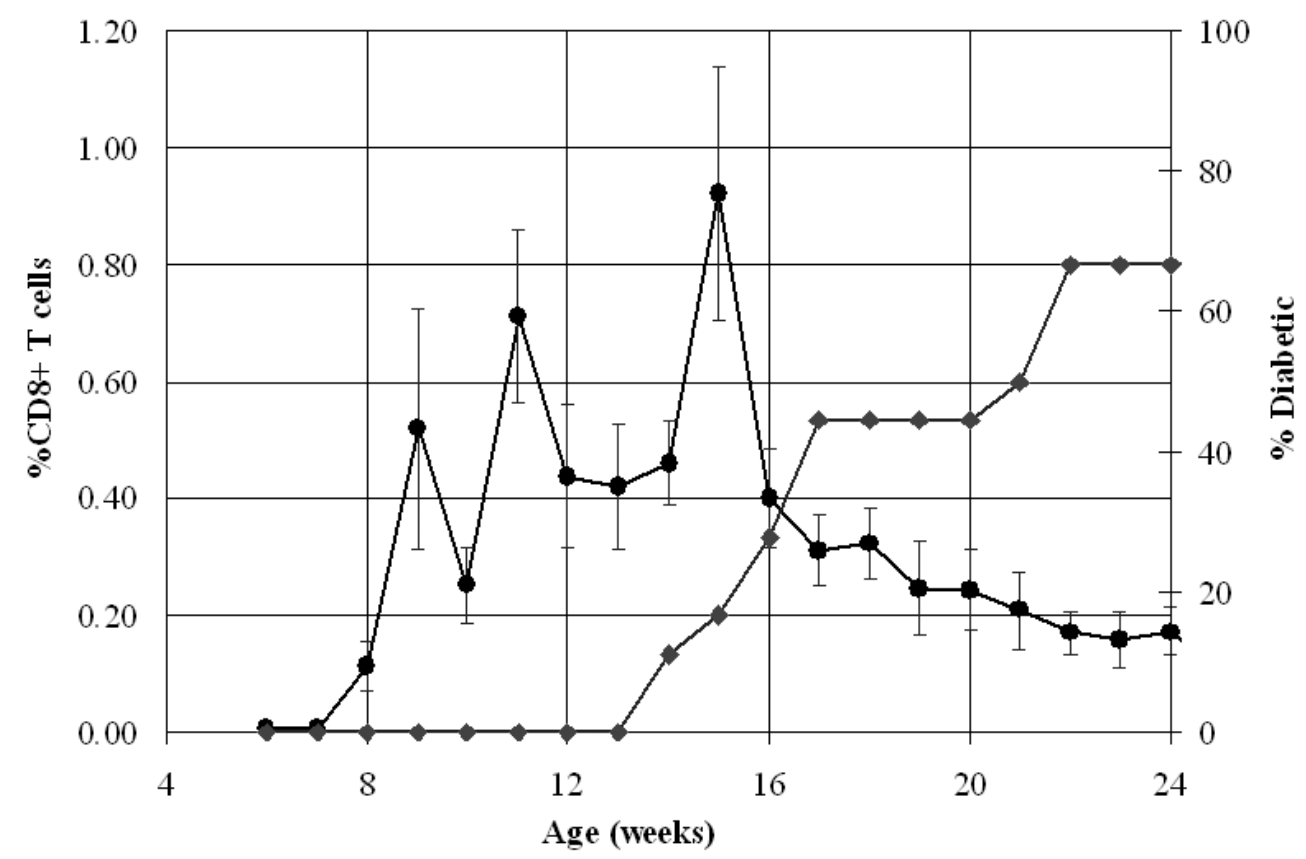

FIG. 1.1. Periodic waves of circulating $T$ cells occur in mice prone to diabetes (NOD mice) in the weeks before the onset of the disease. Data reprinted with permission from authors of [23]. Dark line, circles: T cell level. Grey line, squares: percentage of the animals that became diabetic. Our model accounts for the cyclic waves, but not for the period of initialization in weeks 0-5, when other processes prime the adaptive immune system.

Using such tetramer staining experiments, it was shown by Trudeau et al. [23] that the level of auto-reactive $\mathrm{CD}^{+} \mathrm{T}$ cells is detectable in the pancreatic islets in 4-5 week old NOD mice, and at elevated levels by weeks 11-14. Correlated with this rise, populations of $\mathrm{T}$ cells circulating in the blood are also noticeably elevated over weeks 4-16 of age, before the high blood-sugar symptoms of diabetes occur. Surprisingly, the levels of these cells do not simply rise monotonically as the disease progresses, but rather, undergo dramatic fluctuations over this time frame, as shown above in Fig 1.1.

Not all NOD mice develop diabetes, but presence of these cyclic T cell waves in a given animal predicts that it will become diabetic. Data for each one of the mice were aligned at the time of onset of high-blood sugar symptoms, so that the time axis could be "normalized" before combining and averaging. These 
pooled data show three peaks in the level of $\mathrm{T}$ cells starting at about 8 weeks of age, and declining from about 16 weeks. The amplitude of the cycles increases over this time, and a slight increase in the period is also visible. The fact that Fig 1.1 was produced experimentally as an average of data for many mice suggests that there is some robustness in the cycling (as well as in its period) in NOD mice. These mice are all genetically identical, which means that parameters typical of their physiological and immunological processes are likely very similar (with some possible exceptions due to environmental effects). Trudeau et al. speculated that each of these cycles represent "a round of proliferation of autoreactive $\mathrm{T}$ cells undergoing avidity maturation" [23], but the details of the underlying mechanism were not explored. This exploration is the subject of our paper.

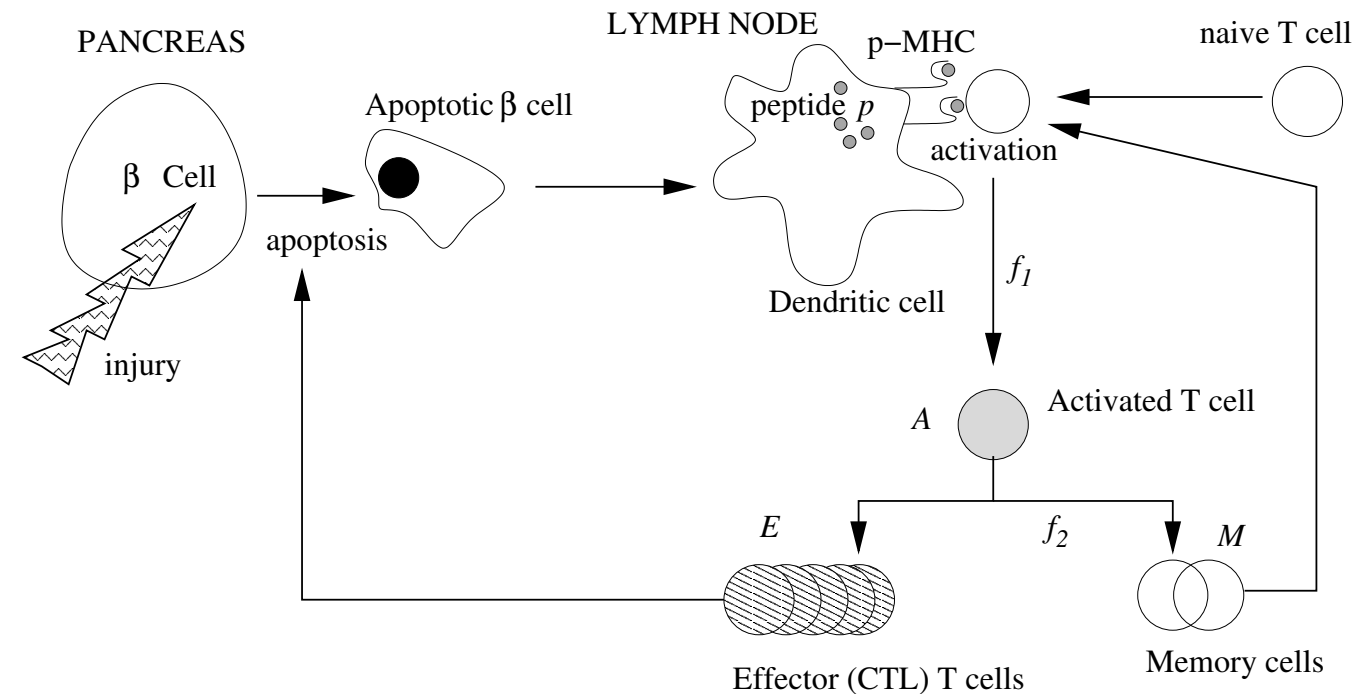

FIG. 2.1. Scheme of the model. Programmed cell death (apoptosis) of pancreatic (insulin producing) beta-cells generates self-antigen peptide ( $p$ ). In the pancreatic lymph nodes, this peptide is presented as part of cell-surface complexes (peptide$M H C$, or $p-M H C)$ on antigen presenting cells called dendritic cells. The amount of $p$-MHC presented affects the activation and the differentiation of naive $T$ cells into memory cells (for self-renewal) and into effector cells (cytotoxic $T$ cells, or CTL's) that seek and kill beta cells. This leads to more peptide exposure and results in positive feedback that eventually culminates in autoimmunity and type 1 diabetes.

2. Background for the model. Our main hypotheses stem from a recent model by Marée et al. [14] that addressed the dynamics of $\mathrm{T}$ cells and peptide. In the latter paper, the focus was on artificial peptide used to treat the disease in a therapy similar to vaccination. It was shown that the competition of $\mathrm{T}$ cell clones during peptide treatment could explain some of the puzzling dose-response behaviour of the treatment, and predict its success or failure. In their discussion, Marée et al. [14] speculated that the increase in level of peptide antigen that results from beta cell killing could be a feedback that explains the periodic waves of $\mathrm{T}$ cells observed by [23]. However, this idea has not yet been tested rigorously in a mathematical setting. We use some of the formalism and lessons learned in that model to investigate cyclic dynamics seen in [23]. We will show that an explanation for such dynamics is already inherent in the framework of the model of [14], or slight variations thereof.

Figure 2.1 summarises the essential ingredients of our model. As shown, the process might be initiated by some injury or infection of beta cells, or by the normal wave of programmed cell death (apoptosis), not shown. Fragments of apoptotic cells are processed and presented as p-MHC on dendritic cells in the lymph nodes, and naive $\mathrm{T}$ cells interact with these complexes. It is known that the level of peptide presentation (i.e., amount of p-MHC) and the affinity of the $\mathrm{T}$ cell receptors for the peptide determines whether a $\mathrm{T}$ cell encountering the antigen presenting cell will become activated to proliferate $[4,6,16]$. When naive $\mathrm{T}$ cells are activated, they proliferate to produce about 60 effector cells and about 1-4 memory cells [8]. Memory cells have a low turnover rate. They are able to undergo reactivation in response to antigen and to proliferate again, replenishing the pool of $\mathrm{T}$ cells. By killing beta cells, the effector $\mathrm{T}$ cells lead to a positive feedback on the amount of peptide produced, and hence on further activation of $\mathrm{T}$ cells. The life-time of the effector $\mathrm{T}$ cells is about 3 days $[5,7,22]$ versus about 100 days for memory cells.

The level of peptide influences two important aspects of the process described above. First, the rate of activation of $\mathrm{T}$ cells depends on peptide level. Second, the fraction of daughter cells that are memory cells versus those that are effector cells is also peptide dependent. Experimental evidence [11, 17] points 
to the fact that, at high peptide doses, too few memory cells are produced. (This is termed "clonal exhaustion"). Following [14], we assume that the fraction of of naive and memory T cells activated is given by a sigmoidal increasing function, $f_{1}(p)$, whereas the fraction, $f_{2}$, of daughter cells of activated T cells that become memory cells decreases sigmoidally as peptide increases. We also chose $f_{1}$ and $f_{2}$ to be Hill functions, i.e., rational functions with powers of degree $>1$ (the degree is called the Hill coefficient, see Section 3.2.)

In Figure 2.2, we show a simplified scheme, outlining our basic assumptions for the model: A fraction $f_{1}$ of incoming naive $T$ cells become activated $(A)$; a fraction, $f_{2}$, of their offspring are memory cells $(M)$, and the rest, $1-f_{2}$, are effector cells, $(E)$. Memory cells can be reactivated (same peptide-dependent fraction, $f_{1}$, as incoming naive $\mathrm{T}$ cells). The effector cells cause death of beta cells, $(B)$, which, in turn, creates the peptide $(p)$. The peptide level affects both $f_{1}$ and $f_{2}$.

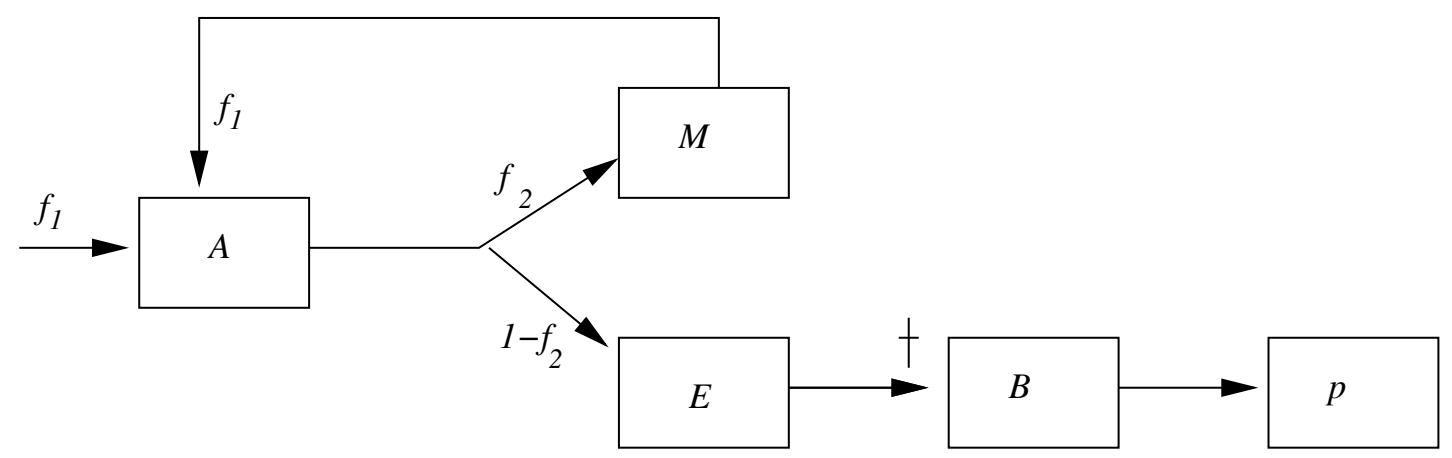

FIG. 2.2. Simplified model scheme, showing the main variables considered: $A, E, M$ are the number of activated, effector, and memory $T$ cells. $B$ denotes beta cells, and $p$ is peptide. The two peptide-dependent functions are the fraction of $T$ cells activated, $f_{1}$, and the fraction of memory cells produced, $f_{2}$. (The feedback from peptide to these has been omitted in the diagram for clarity). The $\dagger$ represents killing of beta cells by effector $T$ cells.

\section{The Model.}

3.1. Assumptions. The following assumptions enter the model

1. We do not at this stage consider the distinct compartments of blood, pancreas, and lymph nodes. Since the dynamics of interest take place over many weeks, whereas the trafficking between these compartments takes place on the time scale of hours, we approximate all variables as densities or concentrations in a single, well-mixed compartment.

2. We do not model the pathogenesis of the disease over the first 4-5 weeks. At this early stage, it is likely that the innate immune system (e.g., macrophages) may set up conditions that eventually give rise to priming of $\mathrm{T}$ cells. See [13] for an analysis of that stage.

3. We assume that effector cells are terminal. (Some controversy exists about whether they give rise to some memory cells.) We also investigated a model in which memory cells are progeny of effector cells and found essentially similar results.

4. We do not discuss the competition of many distinct "clones" of T cells for sites on antigenpresenting cells or for p-MHC [14]. We model only the development of one dominant clone.

5. We assume that material from dead beta cells produces self-antigen peptide at a linear rate, and that this peptide is presented proportionally as p-MHC on the dendritic cells. In [14], this p-MHC level was denoted $m_{t}$ and modeled as a quantity in quasi-steady state (QSS) with peptide and MHC molecules. Here we simplify such details.

6. We assume that once beta cells are gone, the production of the autoantigen ceases, and the immune response stops, since $\mathrm{T}$ cell activation does not occur in the absence of peptide.

3.2. Model equations. Our full model consists of the following set of ordinary differential equations (ODE's):

$$
\begin{aligned}
\frac{d A}{d t} & =(\sigma+\alpha M) f_{1}(p)-\left(\beta+\delta_{A}\right) A-\epsilon A^{2}, \\
\frac{d M}{d t} & =\beta 2^{m_{1}} f_{2}(p) A-f_{1}(p) \alpha M-\delta_{M} M, \\
\frac{d E}{d t} & =\beta 2^{m_{2}}\left(1-f_{2}(p)\right) A-\delta_{E} E,
\end{aligned}
$$




$$
\begin{aligned}
\frac{d p}{d t} & =R E B-\delta_{p} p, \\
\frac{d B}{d t} & =-\kappa E B
\end{aligned}
$$

where $A(t), M(t), E(t)$ are the population levels of activated, memory, and effector $\mathrm{T}$ cells at time $t$, $p(t)$ is the peptide level, and $B(t)$ is the population of remaining beta cells. For the peptide-dependent functions we take Hill functions,

$$
\begin{aligned}
& f_{1}(p)=\frac{p^{n}}{k_{1}^{n}+p^{n}}, \\
& f_{2}(p)=\frac{a k_{2}{ }^{m}}{k_{2}^{m}+p^{m}} .
\end{aligned}
$$

with $m, n>1$. The parameters $k_{1}>0$ and $k_{2}>0$ in Eqns. (3.6) and (3.7) denote typical levels of peptide at which the response of these functions is half-maximal, and $0<a<1$ is the maximal value of $f_{2}(p)$. Note that $f_{1}(p)$ is monotonic increasing whereas $f_{2}(p)$ is monotonic decreasing with $p$. In Eqs. (3.1)-(3.3), all $\mathrm{T}$ cells represent members of clones whose specificity to beta-cell peptide is high. In Eqn. (3.1), $\sigma$ is the rate that naive $\mathrm{T}$ cells enter the circulation from the thymus. The fraction of incoming naive and memory cells that become activated is governed by the peptide-dependent sigmoidal function $f_{1}(p),(\alpha$ is a factor that represents the higher rate of activation of memory cells relative to naive cells). The rate of decay of $A, \delta_{A}$ is augmented by a term for competition, $\epsilon A^{2}$, as discussed in [14]. Activated cells progress to a differentiated stage at rate $\beta$. They then proliferate by a series of cell-doublings to produce $2^{m_{2}} \approx 60$ effector cells, and $2^{m_{1}} \approx 3-4$ memory cells. The commitment to development into these two types of daughter cells depends on peptide according to the decreasing sigmoidal function $f_{2}(p)$. Effector cells are terminal, and have a shorter half-life than memory cells $\left(\delta_{M}<\delta_{E}\right)$.

Equation (3.4) depicts our simple assumption about production and clearance of peptide: the level of "peptide," $p$, is produced with mass-action kinetics when effector cells kill beta cells (at rate $R$ per effector per beta cell) and cleared with linear kinetics at rate $\delta_{p}$. Recall that clearance of dead beta-cells and their fragments by macrophages is defective in NOD mice $[12,18,19]$, and this defect can theoretically lead to the early chronic inflammation that initiates the priming of $\mathrm{T}$ cells [13]. Therefore, it is of interest to ask whether this same defect can also account partly for the dynamics of $\mathrm{T}$ cells at this later stage of the disease. We investigate this further on.

We use the simplest possible model for decay of beta cells due to killing by effector $\mathrm{T}$ cells in Equation (3.5). The parameter $\kappa$ denotes the rate of killing per effector cell. We ignore the (limited) ability of beta cells to regenerate, and the very slow aging and turnover rate of beta cells in the healthy individual. Currently, the extent to which beta cells can self-renew after immune attack is still under investigation, and this process is likely to occur on a slow timescale. For this reason, we did not explicitly include this in the model at this stage.

3.3. Model equations for a reduced QSS system. Our analysis begins with a reduction of the full system of equations (3.1-3.5) to a simpler model using separation of time scales. First, we argue that the timescale of peptide dynamics - hours - is faster than any of the timescales of cell dynamics - days and weeks - justifying a quasi-steady state assumption (QSS) on the peptide. Hence, we set $d p / d t=0$ in the model, so that $p=\left(R B / \delta_{p}\right) E$.

The model then consists of Eqns. (3.1), (3.2), (3.3) and (3.5). The functions $f_{1}, f_{2}$ now depend on $E$ and $B$ via the QSS peptide expression. We refer to this as the reduced QSS model. Our first step was to explore this model computationally. To do so, we had to estimate parameters and consider appropriate scaling. Our steps and results are described below.

3.4. Parameter estimates, scaling arguments, and computations. Based on nonlinearities (in the functions $f_{1}, f_{2}$ ), the model consisting of Eqns. (3.1-3.5) can have a range of interesting behaviours. As we are interested in the possible biological and medical applications of this model, it is essential to study its behaviour within a biologically reasonable range of parameter values. Almost all parameters in the model were based on experimental information previously compiled by Marée et al. [14]. Some exceptions include parameters associated with beta-cell killing and peptide production, as these were not considered in the previous treatment. To avoid lengthy diversion into the details, we concentrate all details of the parameter estimates in the Appendix. The meanings, units, and values of the parameters are presented in Table B.1. The level of cells of type $A, E, M$ vary on a range of several orders of magnitude. As we wanted to present these all on the same plot, we scaled these population densities 
by the appropriate powers of ten. Scaling arguments are also given in the Appendix. We left the time variable in units of days, to emphasize the period and timing of the cycles that we obtained.

Simulations of the dynamics were carried out in Matlab. Initial conditions were chosen to depict some (preexisting) stimulus to the immune system stemming from earlier stages of the disease (e.g., as speculated in [13]). Bifurcation diagrams were composed with the auto feature of XPP, freely available software written by G Bard Ermentrout ${ }^{1}$. Unless otherwise indicated, all simulations use the basic core set of parameter values, as shown in Table B.1.

4. Results. Starting any simulation with the healthy state as initial condition, i.e., $A=M=E=0$, $B=1$, (and thus also $p=0$ ) clearly results in continued health, since this point is a steady state of the system. Moreover, the stability of this equilibrium implies that even some (sufficiently small) perturbation rapidly returns to this state. Hence to get any immune dynamics of interest in our model, the system should be initiated with some $\mathrm{T}$ cells already "primed". Typically, we start simulations with $A=0.5, M=0$, and $E=1$. This state ensures that effector cells are present to lead to peptide production, and that activated $\mathrm{T}$ cells are available to renew that pool of effectors. Other initiation values are possible, depending on parameter settings (discussed later). This prototypical set of values represents the outcome of earlier events that our model is not describing (but see, e.g., [13] for possible description.)

Not all NOD mice develop diabetes. Therefore, any model for this disease also has to account for the fact that some initial stimuli will be resolved without full-blown autoimmunity. We first discuss this baseline control for the model. Running the reduced QSS model from an initially "primed" state, with default parameter values gives rise to the behaviour shown in Figure 4.1; that is, an initial elevated level of effector and memory $\mathrm{T}$ cells is resolved, after some time, and the immune response ceases. This corresponds to resolution of the immune attack with no autoimmunity even though the immune system has been provoked to respond. The beta cell population decreases by $40 \%$ during the immune attack. Since our model does not address replenishment of the beta cells by reproduction or stem cell differentiation, the beta cell mass remains constant after this isolated immune response.

When the initial conditions include more elevated levels of activated $\mathrm{T}$ cells (with all other parameters left as is), oscillations can appear, as shown in Figure 4.2. As in Trudeau et al. [23], three peaks with increasing amplitude of effector $\mathrm{T}$ cells occur over days 30-80, with period approximately 3-4 weeks as in the experimental data. This run is in close agreement with the data for mice that develop full-blown diabetes, as shown in Fig. 1.1.

We can understand intuitively how such cycles occur by reasoning as follows: In our model, Equation (3.5) leads to decay of beta cells whenever effector cells are present. Due to the assault on beta cells by the $\mathrm{T}$ cells (specified by our choice of initial conditions), peptide level increases, $\mathrm{T}$ cells are activated, and effector cells are formed. However, once peptide rises to a high level, memory cell production is turned off (as $f_{2}$ decreases with $p$ ). Thus, replenishment of activated $\mathrm{T}$ cells, drops and subsequently also $E$ therefore declines and is not renewed. Once the effector cells decline, new peptide is hardly produced. It is gradually cleared and eventually reaches a low level that is then consistent with memory cell production. This then stimulates production of new activated T cells, and the cycle repeats. Periodic peaks and troughs continue until beta cells are depleted, and then no more peptide is formed, and $\mathrm{T}$ cell activation stops altogether. At this stage, since beta cells are gone, full blown diabetes sets in, and the immune response decays to its trivial equilibrium. This reasoning is plausible, but relies on an appropriate combination of parameters governing rates of depletion and renewal of the various cell types.

It is noteworthy that merely by increasing the rate of clearance of the peptide, $\delta_{p}$, we end the tendency of the system to cycle. We ran simulations with elevated values of $\delta_{p}$, and found behaviour similar to that of Fig 4.1 for much broader ranges of initial conditions (results not shown). These results can be taken as indications that in "control" mice whose peptide clearance rate is normal, immune response is less likely to lead to prolonged cycling attack. These results are discussed in more detail later on.

5. Analysis of a reduced model with $B$ as a parameter. To gain a clearer understanding of the behaviour described above, we reduce the four dimensional model simulated above yet further by considering the level of beta cells, $B$, to be a parameter. The onset of diabetes in NOD mice requires about 16 weeks at which time there are very few remaining beta-cells in the pancreas. This indicates that the variable $B$ in the full model acts more like a slowly varying parameter compared to the other variables in the model. We therefore consider a reduction to three variables $(A, M, E)$, and analyse the model behaviour. We then discuss how the gradual decrease of $B$ influences the dynamics of the whole system. The model to be analyzed now consists of the three equations:

\footnotetext{
${ }^{1} \mathrm{XPP}$ is freely available at www.math.pitt.edu/ ${ }^{\sim}$ bard/xpp/xpp.html
} 


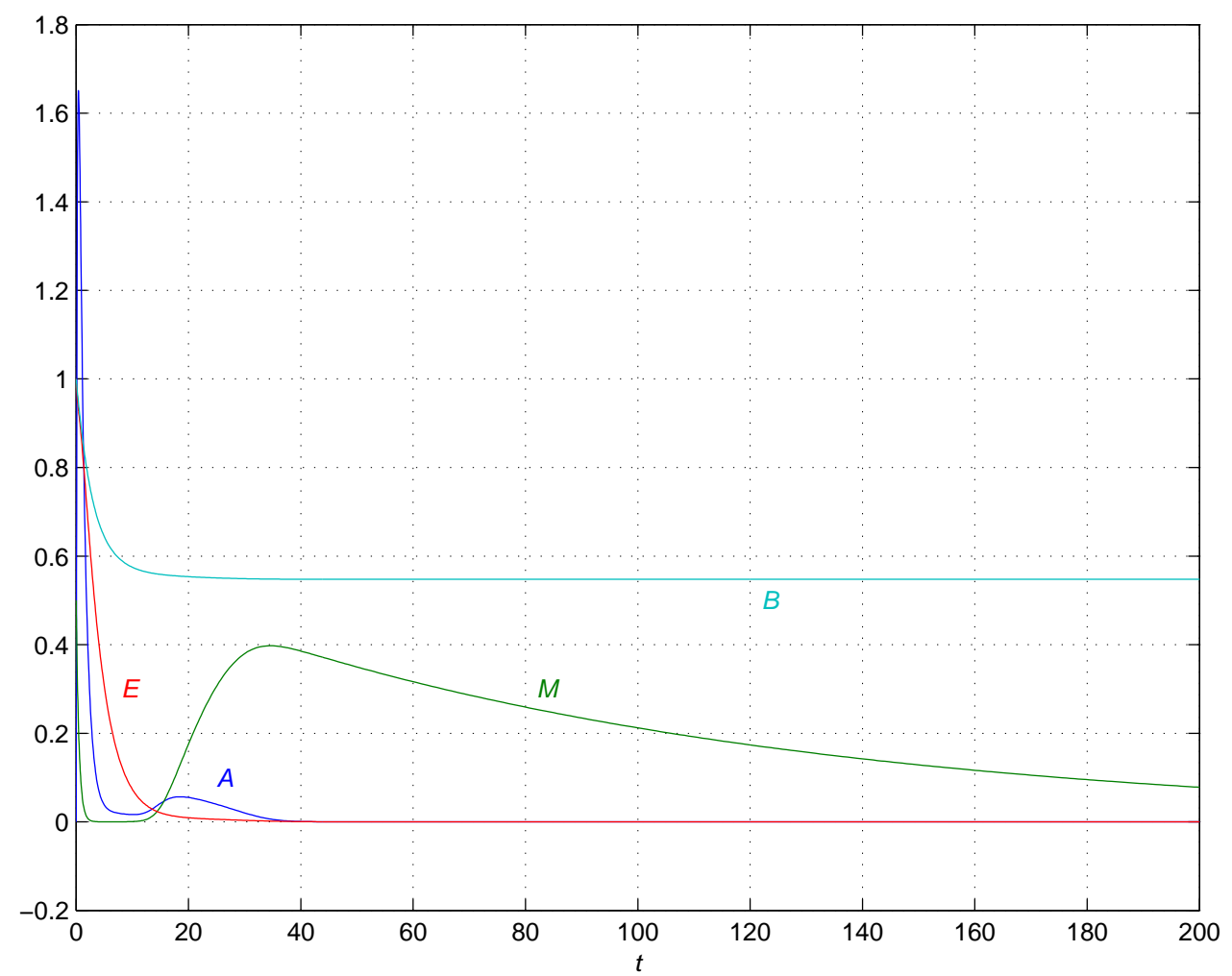

FIG. 4.1. Simulation of the model for NOD mice that do not become diabetic. Number of circulating cells (scaled) vs time (days). Dark blue: $A\left(\times 10^{3}\right.$ cells), Green: $M\left(\times 10^{4}\right.$ cells), Red: $E\left(\times 10^{6}\right.$ cells), light blue: $B$ (fraction of beta cell mass remaining). Simulation uses default ("NOD") parameter values given in Tables B.2 and B.1. For the initial conditions $A=0, M=0.5, E=1, B=1$, the immune response is resolved without chronic disease or cyclic waves.

$$
\begin{aligned}
\frac{d A}{d t} & =(\sigma+\alpha M) f_{1}(p)-\left(\beta+\delta_{A}\right) A-\epsilon A^{2}, \\
\frac{d M}{d t} & =\beta 2^{m_{1}} f_{2}(p) A-f_{1}(p) \alpha M-\delta_{M} M, \\
\frac{d E}{d t} & =\beta 2^{m_{2}}\left(1-f_{2}(p)\right) A-\delta_{E} E,
\end{aligned}
$$

together with Eqns. (3.6) and (3.7), and the QSS peptide expression

$$
p \approx\left(R B / \delta_{p}\right) E .
$$

This three dimensional system of differential equations permits a more complete analysis.

5.1. Steady states and stability properties. The 3D system of differential equations given by Equations (5.1-5.3) has several types of feedback. Peptide level (and therefore effector cell level) leads to positive feedback on $\mathrm{T}$ cell activation via $f_{1}$. Simultaneously, these levels produce negative feedback on the memory cell production via $f_{2}$. When combined, these nonlinear feedbacks lead to the possibility of multiple steady-states, depending on the parameters. Numerical experiments suggest that this mixed feedback system can have from one up to five equilibria.

In the biologically relevant regime of parameters (discussed in the Appendix), we find that there are three equilibria. One of these is clearly the trivial equilibrium $A=M=E=0$. This follows immediately from the fact that $f_{1}(0)=0$. This equilibrium corresponds to a disease free state and is easily shown to be a stable node. The fact that the origin is an attractor means that a small disturbance that provokes the immune system should be resolved, provided it is sufficiently weak.

There also exists a positive equilibrium that corresponds to a state of elevated immune cell levels. In that state, effector $\mathrm{T}$ cells are continuously killing beta cells and this corresponds to an autoimmune attack that eventually leads to diabetes. This equilibrium has various stability properties that depend on the parameters. We discuss this in more detail below. A third equilibrium is a saddle with a twodimensional stable manifold, which for some parameters separates the "healthy" and diseased equilibria. 


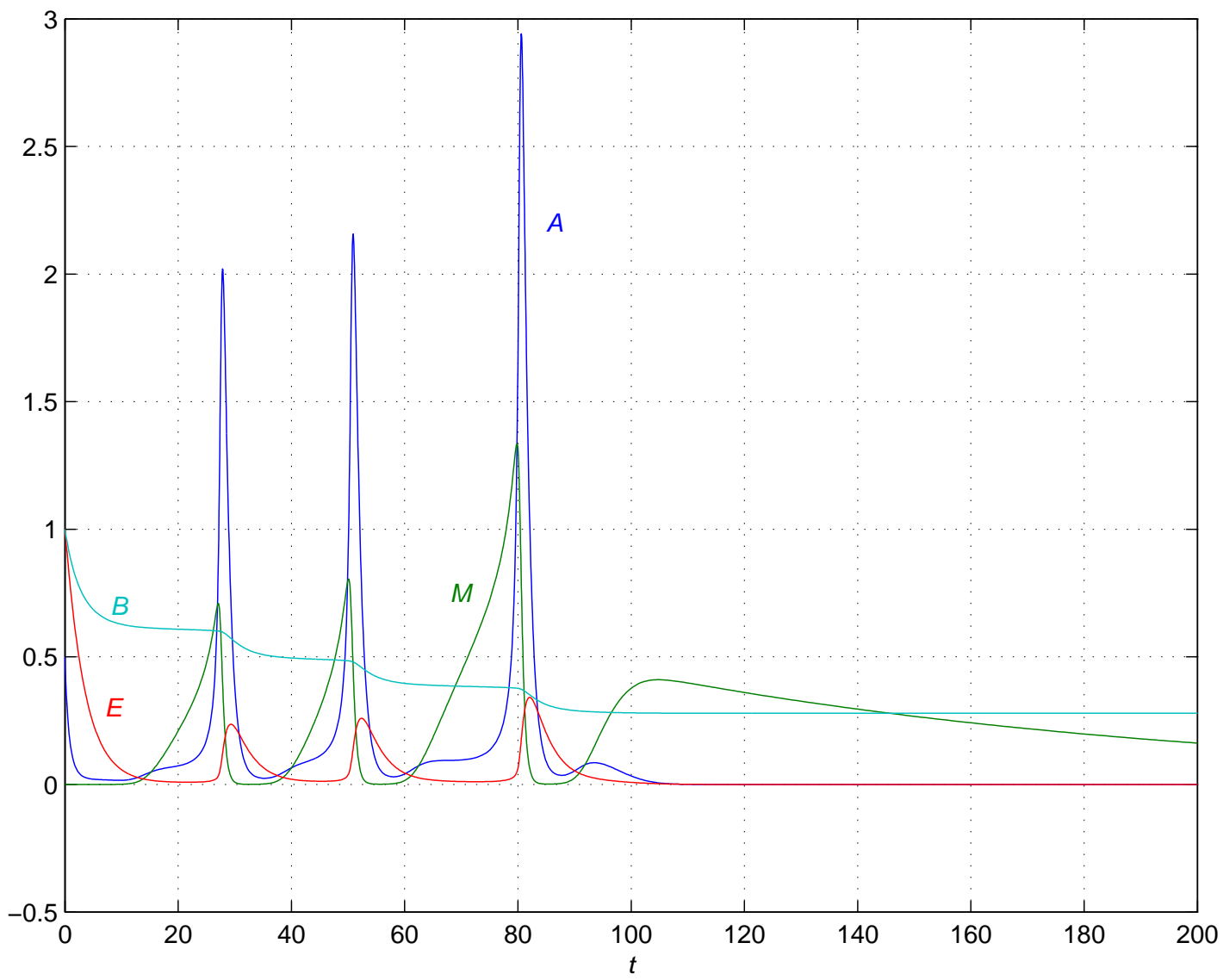

FIG. 4.2. Simulation of the model for NOD mice that do become diabetic (by 80-90 days of age). Default ("NOD") parameter values, and scaling as in Figure 4.1, but with initial conditions $A=0.5, M=0, E=1, B=1$ that evoke the elevated periodic immune response. Dark blue: A, Green: $M$, Red: E, light blue: B. The disease progresses with cycles of $T$ cells that cause waves of beta cell killing, as predicted by the model.

For these parameters, stimuli that fall on the wrong side of this separatrix will be attracted to the diseased equilibrium. For other parameter values, the unstable manifold of the diseased state connects to the stable manifold of the saddle point. In this case, almost all positive initial conditions asymptotically, approach the "healthy" state.

As a specific example of the local analysis, we considered the system of equations (5.1-5.3) with the parameters given in Table B. 1 and $B=1$. Due to the nonlinearities in the functions $f_{1}$ and $f_{2}$, it is not possible to solve explicitly for equilibria. Therefore, we determined steady states, eigenvalues, and eigenvectors numerically, using the software program Maple. We found the following results: The disease-free equilibrium, $\left(\bar{A}_{0}, \bar{M}_{0}, \bar{E}_{0}\right)=(0,0,0)$, is a stable node with the three eigenvalues $\lambda=-1,-0.3,-0.01$. A saddle node at $\left(\bar{A}_{s}, \bar{M}_{s}, \bar{E}_{s}\right)=(0.0116,0.696,0.00116)$ has a two-dimensional stable manifold (eigenvalues $\lambda_{1}=-1.52, \lambda_{2}=-0.0188$ and associated eigenvectors $\left.v_{1}=[1,0.495,0.0245], v_{2}=[1,-68.5,0.107]\right)$ and an unstable manifold (eigenvalue $\lambda_{3}=0.210$ with eigenvector $v_{3}=[1,2.62,0.0589]$ ). Finally, the diseased equilibrium, $\left(\bar{A}_{d}, \bar{M}_{d}, \bar{E}_{d}\right)=(0.119,0.0141,0.0356)$ has a stable manifold (with eigenvalue $\lambda_{1}=-2.37$ and associated eigenvector $\left.v_{1}=[1,-0.108,-0.0414]\right)$. It also has a two-dimensional unstable manifold (eigenvalues $\lambda=0.0129 \pm 0.553 i$ ) that spirals outward toward a limit cycle. From this local analysis, we could see that at each equilibrium, one eigenvalue is significantly more negative than the others. This suggests that there is a globally attracting two-dimensional manifold containing the three equilibria, where the interesting dynamic behavior occurs.

5.2. Bifurcations. We first discuss bifurcations with respect to a relevant parameter, and later assemble the sequence of dynamical behaviours in Fig. 6.2. In the model given by Eqns. (5.1-5.3) we have assumed that the destruction of beta cells occurs on a slow time scale. Thus, the level of beta cells, $B$, makes a natural bifurcation parameter to consider. At the beginning of our simulations, we normalize $B=1$ and set $\delta_{p}=1$. By the QSS assumption for peptide, a gradual loss of beta cells in this model variant is dynamically equivalent to a gradual increase in the peptide clearance rate $\delta_{p}$. (Both parameter 
variations essentially describe the decreasing QSS value, $p=\left(R B / \delta_{p}\right) E$.) We explored this parameter variation using the AUTO option of the software XPP. Figure 5.1 shows the result obtained thereby.

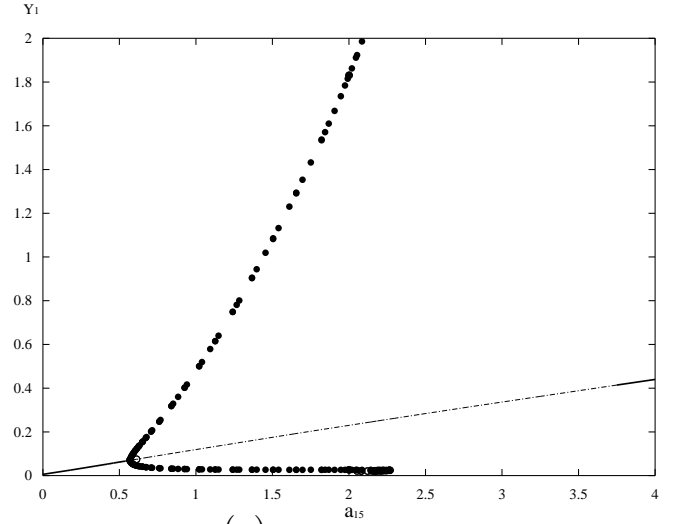

(a)

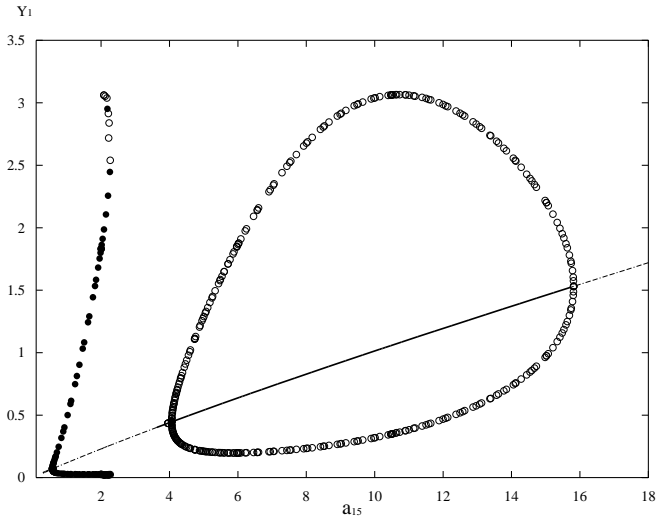

(b)

FIG. 5.1. Bifurcation diagram for the peptide decay rate, $a_{15}=\delta_{p}$ with all other parameters set at their default values, as in Tables B.2 and B.1. The vertical axis is $A$ in units of $10^{3}$ cells. (a) A portion of the diagram, enlarged, shows the typical bifurcation: A Hopf bifurcation occurs at $a_{15}=0.5707$ spawning a stable limit cycle. A homoclinic bifurcation occurs at $a_{15}=2.268$. (b) Further bifurcations on an expanded scale: another Hopf bifurcation (to an unstable limit cycle) occurs at $a_{15}=4.063$. This limit cycle vanishes at $a_{15}=20.28$.

The diagram given in Figure 5.1(a) shows the basic bifurcation behaviour of the model (and uses the default parameters values given in Tables B.2 and B.1. Moving across this diagram from left to right along the horizontal axis represents increasing values of the peptide decay rate $\delta_{p}$, or equivalently, a decreasing level of beta cells, $B$. Close to the leftmost edge, (high $B$, or low peptide clearance rate), we find a stable diseased state (solid line with shallow slope). The "healthy" state, also stable, and the saddle node are not indicated on the diagram. Moving towards the right, leads to a supercritical Hopf bifurcation at $a_{15}=\delta_{p}=0.571$, spawning a stable limit cycle. Here we enter the regime of cyclic behaviour evidenced in Figure 4.2. The diseased equilibrium is then an unstable spiral, as predicted by the local analysis described above. The limit cycle persists, and its amplitude increases as the parameter increases (respectively, as the beta cell level decreases) up to a homoclinic bifurcation at $\delta_{p}=2.268$ (equivalently at $B=0.441$, i.e., when only about $44 \%$ of beta cell mass remains). As seen in our runs, and in the upper branch of this bifurcation line on the zoomed out diagram of Fig 5.1(b), AUTO has difficulty resolving this global bifurcation. We discuss the nature of this dynamical shift further on.

Following the homoclinic bifurcation, the diseased state remains unstable, and the origin is the only global attractor for some range of the bifurcation parameter. Interpreting this bifurcation diagram in terms of normal and reduced levels of (peptide) clearance rates (by control vs NOD macrophages) suggests why the clearance defect itself could make the difference between healthy (control) mice versus diabetesprone (NOD) mice: for example, as seen in Fig 5.1(a), a "control" peptide clearance rate of $\delta_{p}=3$ per day leads to dynamics that always resolve any initial stimulus (returning to baseline where no immune cells persist, since the limit cycle does not occur, and the disease state is unstable) whereas a factor of two decrease to $\delta_{p}=1.5$ per day (representing reduced clearance in NOD mice) puts the same system into the regime of cyclic $\mathrm{T}$ cell waves and autoimmunity.

Reinterpreting this diagram in terms of the gradual decrease of beta-cell mass (from left to right starting from $B=1$ ) explains the following features shared by the data of Fig. 1.1 and the simulation of Fig. 4.2: (1) the increase in the amplitude of the cycles, (2) the fact that the cyclic behaviour stops abruptly (e.g., around days 80-90 in the simulation of Fig 4.2) when the homoclinic bifurcation occurs, and (3) the slight lengthening of the period just before this transition. It also explains why (4) the immune cells then decay to the baseline state $A=M=E=0$. Thus, the bifurcation diagram can help to provide a plausible scenario for a mechanism underlying these dynamics.

As previously noted, immunological systems present a menagerie of curious dynamical behaviours that can be an enticing invitation to the applied mathematician. As our model is nonlinear, other interesting behaviour is to be anticipated. In Fig 5.1(b) we show an expanded scale, with much higher values of the peptide turnover parameter. As seen here, at $\delta_{p}=4.063$ per day, a second subcritical Hopf bifurcation takes place. Thus, for a range of values of $4.063<\delta_{p}<20.28$ per day, the diseased state becomes (locally) stable once more, with a domain of attraction bounded by an unstable limit cycle. All solutions inside this domain will evolve towards the diseased state, whereas outside this domain of 


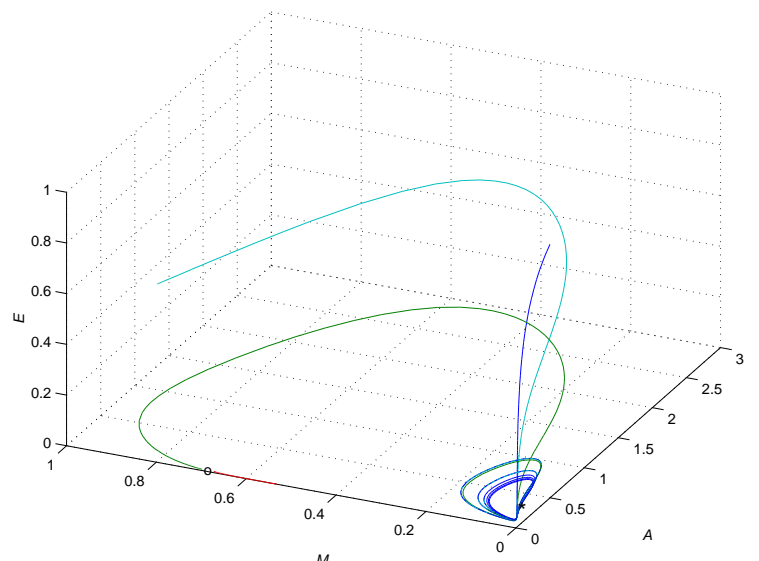

(a)

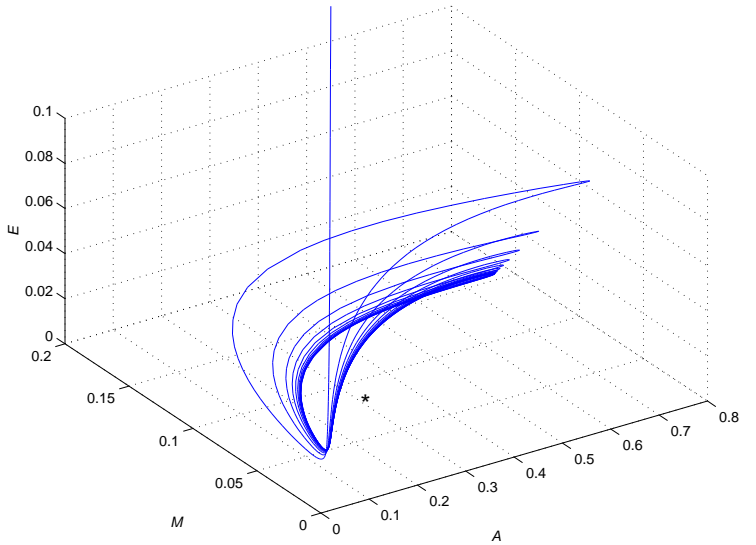

(c)

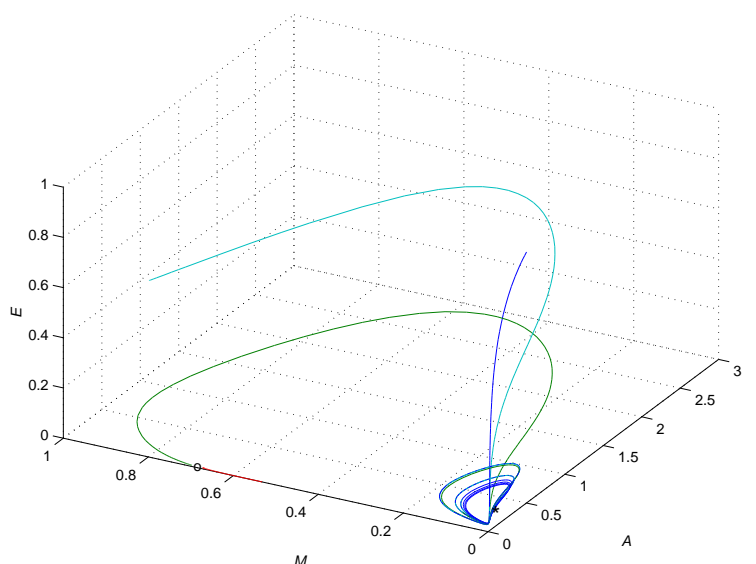

(b)

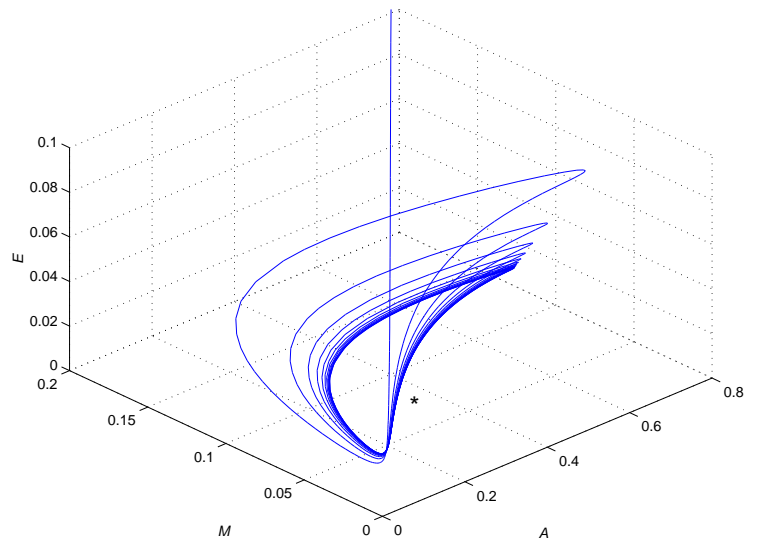

(d)

FIG. 6.1. Two stereograms showing $(a, b)$ the limit cycle and location of the saddle point (marked $\circ)$ and $(b, c)$ the limit cycle and its unstable diseased equilibrium (denoted by $*$ ). Both diagrams were made for the basic model with default parameter values, but with beta cell mass treated as a (constant) parameter.

attraction, solutions eventually lead to the origin. Aside from purely mathematical interest, this diagram suggests that there are as yet other unexplored behaviours in this and other immunological models. On one hand, biologically, this result could be interpreted to mean that increased removal of peptide is not always advantageous (since it can reinstate the stability of the diseased state). On the other hand, the dynamics shown in this expanded parameter regime might be more of a mathematical curiosity than a result that is directly relevant to diabetes in NOD mice.

We investigated a number of other parameter variations and bifurcations (diagrams omitted), starting from the default parameter set. For example, we varied the parameter $a=a_{4}<1$ of the function $f_{2}$. This parameter specifies the maximal fraction of memory cells produced (when $p=0$ ). We found that decreasing $a$ from 1 leads to the homoclinic bifurcation at $a=0.45$. Similarly, for the $\mathrm{T}$ cell competition parameter, the range $0 \leq \epsilon \leq 2.17$ lies within the stable limit cycle regime. A Hopf bifurcation occurs at $\epsilon=2.173$, leading to stability of the diseased state. No homoclinic bifurcation was obtained by varying this parameter. Finally, changing $k_{2}$, the peptide level that corresponds to the half-maximal value of $f_{2}$, gave a stable diseased state when $k_{2}=2$, a Hopf bifurcation at $k_{2}=1.112$, and a homoclinic bifurcation when $k_{2}=0.825$. Due to space constraints, these bifurcation diagrams are not shown.

6. Geometry of the solutions. Figure 6.1 shows two stereograms of the three-dimensional $A M E$ system in the regime of parameters consistent with stability of the limit cycle oscillations. In $(a, b)$, we show the position of the saddle node (close to the $M$ axis) with unstable manifolds in green and red. One branch of the unstable manifold (in red) flows towards the stable disease free state, while the other branch (in green) spirals towards the limit cycle about the disease state. (The 2D stable manifold is not indicated in this figure.) The limit cycle, and two trajectories attracted to it are also shown. In (c,d), a zoomed-in view of the limit cycle is shown. The location of the (unstable spiral equilibrium) diseased state is indicated by a small star.

Figure 6.2(a-d) shows a sequence of diagrams that illustrate the bifurcations and dynamics described 


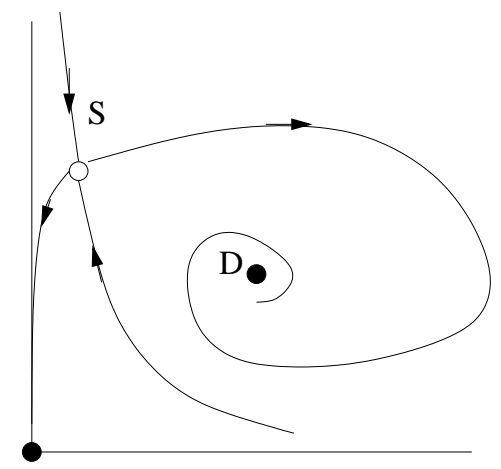

$\mathrm{H}$

(a)

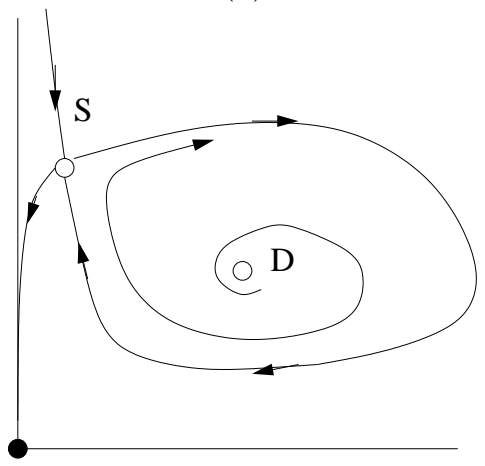

$\mathrm{H}$

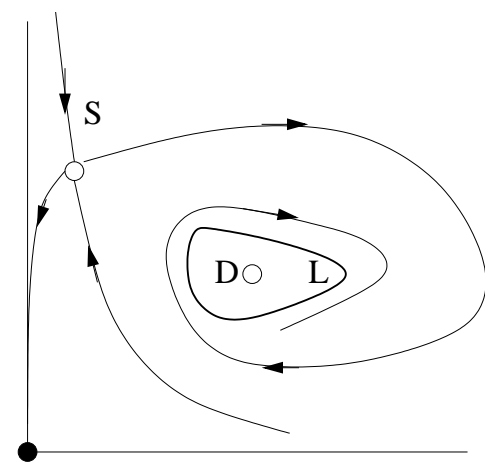

$\mathrm{H}$

(b)

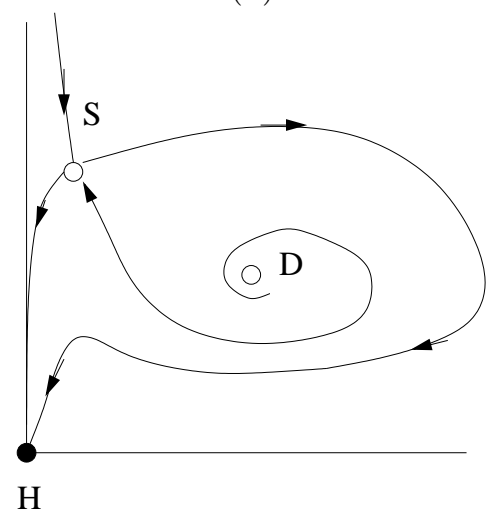

(d)

(c)

FIG. 6.2. This sequence of four sketches illustrate the essential geometry of the dynamics and bifurcations. H: "healthy" state in which there are no circulating immune cells, D: diseased equilibrium; S: saddle node, L: stable limit cycle. Heavy dots indicate stable equilibria, and open dots indicate unstable ones. In (a) an unstable manifold of $S$ winds into the stable spiral at D. In (b), just past a Hopf bifurcation, there is a stable limit cycle to which this manifold is attracted. (c) represents the homoclinic bifurcation. In (d) the unstable manifold of $S$ makes a detour past the unstable $D$, ending at $H$. The state $H$ is always stable. However, the boundary of its basin of attraction is formed by the stable manifold of S. In (d) every initial condition will eventually evolve towards the origin.

in the previous section. We show 2D "cartoons" that give the overall picture (although our $A M E$ system is three dimensional) since it is difficult to numerically simulate the precise parameter set that leads to the homoclinic connection, and equally challenging to represent all stable and unstable manifolds in a 3D plot. As shown in this figure, the origin (heavy dot labeled $\mathrm{H}$ for "healthy") retains its stability and is a local attractor in all cases, but its basin of attraction can vary greatly. In (a) and (b), a separatrix (one branch of the stable manifold of the saddle node S) defines the boundary between those states attracted to $\mathrm{H}$ and others that remain in the positive orthant. In (a), these other points are attracted to the stable diseased state (heavy dot at D), whereas in (b), past a Hopf bifurcation, the limit cycle is attracting. A homoclinic connection (which exists for one specific set of values of the parameters) is illustrated in (c). In (d), states close to the unstable point D may take an "excursion" towards S, but eventually arrive at H. In this case, all solutions of Equations (5.1-5.3), except for a set of measure zero (on the stable manifold of the saddle node), would eventually converge to the disease free state $(\bar{A}, \bar{M}, \bar{E})=(0,0,0)$.

7. Parameter sensitivity. The hallmarks of autoimmune diabetes in NOD mice is that many small perturbations and treatments can "cure" the disease, delay its onset or prevent it from occurring. Thus, the actual (biological) system is sensitive to relatively small changes in essential parameters of the system. In order to explore the sensitivity of the model, we tested how increases and decreases in each of the parameters in Equations (3.1-3.5) affect the dynamics. We used the values of parameters that generated Figure 4.2 as a basic set, and varied each in turn by $10 \%$ up and down. The results are shown in Table B.2.

Recall that the original parameter set is consistent with a stable limit cycle for $B=1$. In Table B.2 we note whether the dynamics obtained by a given parameter change has moved the system in the direction of the homoclinic $(\rightarrow)$ or the Hopf bifurcation $(\leftarrow)$, or, in some cases, beyond those bifurcations. (Arrows are indications of shifts along the type of bifurcation shown in Figure 5.1 (a).) We also indicate the 
number of peaks observed between $t=0$ and the time at which the homoclinic bifurcation occurs. It can be seen that changing some parameters, (e.g., $n, m, k_{2}, k_{1}$ of the peptide-dependent response functions $f_{1}, f_{2}$ ) has a large effect on the number of cycles that occur, increasing the number of peaks up to 910. These parameters control the location of the "activation switch" and the switch in commitment to memory versus effector cells with respect to peptide level. The parameter $\beta$ and the number of memory cells produced, $2^{m_{1}}$, also has a dramatic effect on the behaviour. Other variations, e.g., $\delta_{M}, a, \epsilon$ have a very minor effect.

It is interesting to note that certain slight parameter shifts place the system beyond the homoclinic bifurcation, leading to global stability of the origin (as in Fig 6.2d). This includes a 10\% decrease in the rate of memory cell reactivation, $\alpha$, or memory cell production, $a$, or a $10 \%$ increase in the peptide clearance rate, $\delta_{p}$, or the effector $\mathrm{T}$ cell death rate, $\delta_{E}$ (entries in Table B.2 marked with $\mathrm{S}, \rightarrow$ ). Making these adjustments takes the system out of the cyclic regime and restores global stability of the "healthy" state at the origin.

Here we venture to speculate on implications to the disease itself, and possible treatments. One can envision medical interventions that are designed to affect one or another of the parameters mentioned above in patients with known genetic tendency to autoimmunity. If any of these parameter change(s) could be made before beta cell mass is destroyed, the immune attack could be resolved or prevented. Alternately, if cycles of circulating $\mathrm{T}$ cells are observed, treatments could be applied to knock the system out of its destructive cyclic regime, back to the baseline state. The most effective treatment would be one that targets any of the more sensitive parameters in our model. Because our model is fairly simplistic, it is premature to draw firm conclusions about optimal therapeutic strategies. However, studying parameter sensitivity and bifurcations of more detailed and more realistic models for this disease (or other autoimmune disorders) could possibly lead to new therapeutic strategies. Clearly, in the context of a mathematical model, one can also identify and possibly avoid unforseen complications (e.g., the unstable limit cycle regime in Fig 5.1b) where the disease state regains stability in another range of the parameter $(\mathrm{s})$.

8. Other variants of the model. We considered several variants of the model that incorporated other features or relaxed certain assumptions. First, we considered a model in which memory cells are offspring (rather than sisters) of effector cells. (In that model, a function like $f_{2}$ represented the probability that an effector cell differentiates into a memory cell.) Similar behaviour was obtained in a narrower range of parameters. As this scheme of differentiation is less widely accepted, we here omit the details.

The immune response has several inherent delays. After beta cells die, it takes around 8 hrs to one day for their fragments to be collected, transported to the pancreatic lymph node, processed, and presented by antigen presenting cells. Once $\mathrm{T}$ cells are activated, it take a further 2-3 days for proliferation and production of effector cells. This means that an immune response can take 4-6 days from time of stimulus. We explored some of the effects of delay in the system, by investigating variants of the model that had one or two delays. We found similar dynamics, within a slightly shifted set of parameter regimes. Results were similar to figures previously displayed and are here omitted.

We briefly explored competition of various $\mathrm{T}$ cell clones, to determine how competition between different peptide-dependent cells could affect the dynamics. We found that similar clones tend to cycle together, and that competition was not a major force in the cyclic dynamics. The details are omitted.

9. Experimental tests of the model. This model has been informed by previous theory [13, 14], supplemented by experimental observations. In turn, it suggests new experiments that can be used to verify or refute its conclusions.

First, the model predicts a specific sequence of events, with peaks in memory cells preceding peaks in activated $\mathrm{T}$ cells, preceding peaks in effector cells (as shown in Fig. 4.2). Further, the model predicts that during these cycles, one should be able to observe cycles of apoptotic beta cells in the pancreas (since killing by effector cells occurs via apoptosis). If the presence or sequence of cell types follows some other trend, our model would have to be revised.

Second, the model predicts outcomes of specific interventions. For example, once NOD T cell cycles are observed, poisoning some fraction of their macrophages by administering silica, a known poison for such cells (i.e., reducing the innate ability to clear dead beta cell material and hence reducing $\delta_{p}$ ) should decrease the amplitude of the cycles as well as the period of the cycles (see parameter sensitivity, Table B.2). A dose response of this "macrophage poison" versus dynamical behaviour would show successively decreased cycle amplitude (see also bifurcation diagram 5.1a) for the dependence of cycle amplitude on $a_{15}=\delta_{p}$ ). Alternately, treatments that enhance macrophage clearance of apoptotic ma- 
terial (if possible) could, at sufficient dose, stop the cycles, and retard the development of the disease. A number of similar interventions are predicted by parameter sensitivity. While we cannot expect that we have captured all NOD parameters accurately in this preliminary model, general trends "towards" or "away from" the Hopf or homoclinic bifurcation predicted by the various changes in basic parameters should be indicative of the accuracy or fallibility of the assumptions on which the model is based. Some (but not all of these) are experimentally feasible. Future work with experimental colleagues will address such issues in an experimental setting.

10. Discussion. Our main conclusion in this paper is that cyclic dynamics can arise spontaneously in the immune response leading up to type 1 diabetes, at least under conditions typical of the susceptible (NOD) mice. This fact was conjectured in [14] as a possible outcome of the interplay between the effector $\mathrm{T}$ cells killing the insulin-producing beta-cells, and the feedback from self-antigen produced when those cells are killed. We confirmed this conclusion, by extending the model in [14] to include the death of beta cells, and the accumulation of the antigen that results. Our cyclic dynamics (Figure 4.2) is similar to the experimentally observed cycles (Fig 1.1) in three important ways: (1) It shows cycles of increasing amplitudes, (2) The interpeak time length increases slightly and (3) the cycles stop, and the levels of T cells drop around 16-18 weeks. (At this point, the mouse becomes diabetic in the experimental system.) This behaviour was obtained in a regime of parameters that is based mainly on values assembled from the experimental literature in [14].

We showed that one explanation for these oscillations, illustrated by our model is as follows: beta cell killing produces large quantities of self-antigen peptide, expanding the population of effector cells at the expense of memory cells. This creates a gap in self-renewal of the $\mathrm{T}$ cells that leads to a pause in their reproduction, and reduced effector levels for killing. After a suitable interval, when peptide is cleared, the memory cell production is reinstated and the cycle begins once more. The gradual loss of beta cell mass limits the number of cycles that can occur (to three, in the case of NOD mice). The cyclic dynamics are found for a wide range of parameter values, provided the peptide-dependent functions that control $\mathrm{T}$ cell activation, $f_{1}$, and memory cell production, $f_{2}$, ramp up (respectively down) as peptide level increases. Since the immune system is highly complex, with many feedbacks between cells, chemicals, and tissues, it is possible that other explanations for cycles can be equally compelling. For example, recent work by an experimental collaborator (P Santamaria, U Calgary) has focused on the role of regulatory $\mathrm{T}$ cells and their cytokine IL-2. Positive and negative feedback that is emerging in these experimental investigations will provide future opportunities for modeling and analysis using the tools of nonlinear dynamics.

The main contribution of our study is to explain the mechanism underlying the observed cycles by studying the nonlinear dynamics of the extended model and uncovering its bifurcations. This aspect of our work is particularly apt for readers of SIAM, some of whom may not yet be aware of rich dynamics in immunology. We showed that all three of the observations listed above can be explained as gradual shift of a parameter (the mass of beta-cells remaining) during the course of the immune attack by effector $\mathrm{T}$ cells. This gradual shift moves the system from a regime in which there is a stable limit cycle towards a homoclinic bifurcation. The amplitude of the limit cycle expands very quickly just before this bifurcation, and its period increases (theoretically up to infinity) at the homoclinic connection itself. Beyond that, all points are attracted towards the origin, i.e., the levels of $\mathrm{T}$ cells drop.

We found that the number of peaks that occur in the model shifts when certain parameters are changed (by 10\%). The most sensitive parameters are those appearing in the functions $f_{1}$ and $f_{2}$, but it is unlikely that these are easy to manipulate in an experimental system. As our model is the simplest possible variant of [14] that produces cycles, this sensitivity to parameters may be a price paid for omitting other regulatory features of the immune system. On the other hand, the sensitivity to parameters also suggests numerous experimental tests of our predictions that could, in principle validate or falsify the model. Such tests will be under consideration in future work on this problem. In the original model of [14], competition between various clones of $\mathrm{T}$ cells for sites on antigen presenting cells was considered as an important determinant of dynamics. Here, in preliminary investigations of competition of two clones, we found that clones tended to cycle synchronously. Future work will also address the effect of competition in greater detail.

Our model did not address any of the spatial or compartmental aspects of the immune response. For example, we also did not consider the details of trafficking of $\mathrm{T}$ cells between blood, lymph nodes, and tissue. Some of the detailed movement and interactions of $\mathrm{T}$ cell with dendritic cells in lymph nodes is currently being modeled in conjunction with experimental observations by the group of de Boer and Marée (Utrecht, The Netherlands). These insights will inform future models in immunology, including extended models of autoimmune diabetes.

Finally, our model suggests that there are two distinct outcomes in an autoimmune attack typical of 
type 1 diabetes: (1) The immune attack clearly subsides once the beta cells have been depleted, but here the outcome is full-blown diabetes. This explains observations in NOD mice, but therapeutically, it is an outcome to be prevented. (2) More intriguing, any parameter change that shifts the system beyond its homoclinic bifurcation would also end the immune attack. This can happen through the process of "clonal exhaustion": i.e, so much peptide is presented that memory cell production is turned off completely. It could also happen through arrest of activation, where so little peptide is presented that $\mathrm{T}$ cells no longer become activated. In either case, if this happens before a significant fraction of the beta cells have been killed, it could provide a "cure" that resolves the autoimmunity without diabetes. Here we have hinted at several parameters that could have precisely this type of effect. This suggests that studying more detailed and hence more realistic variants of this model could indicate possible therapeutic strategies, by highlighting which parameters give promising leads for medical targets.

Acknowledgements. LEK is funded by the Juvenile Diabetes Research Foundation and by the Mathematics of Information Technology and Complex Systems (MITACS), Canada. We thank A.F.M. Marée, Eric Cytrynbaum, and members of Beta-CAAN (P. Santamaria, D. Finegood, B. Verchere, J. Dutz, D. Coombs, A Khadra) for helpful discussions and comments.

\section{REFERENCES}

[1] A. Amrani, P. Serra, J. Yamanouchi, J. D. Trudeau, R. Tan, J. F. Elliott, and P. Santamaria, Expansion of the antigenic repertoire of a single T cell receptor upon T cell activation., J. Immunol., 167 (2001), pp. 655-666.

[2] A. Amrani, J. Verdaguer, P. Serra, S. Tafuro, R. Tan, and P. Santamaria, Progression of autoimmune diabetes driven by avidity maturation of a T-cell population., Nature, 406 (2000), pp. 739-742.

[3] J. A. M. Borghans, A. J. Noest, And R. J. De Boer, How specific should immunological memory be?, J. Immunol., 163 (1999), pp. 569-575.

[4] J. A. M. Borghans, L. S. Taams, M. H. M. Wauben, and R. J. De Boer, Competition for antigenic sites during T cell proliferation: a mathematical interpretation of in vitro data., Proc. Natl. Acad. Sci. U.S.A., 96 (1999), pp. 10782-10787.

[5] R. J. De Boer, M. Oprea, R. Antia, K. Murali-Krishna, R. Ahmed, and A. S. Perelson, Recruitment times, proliferation, and apoptosis rates during the CD8 ${ }^{+}$T-cell response to lymphocytic choriomeningitis virus., J. Virol., 75 (2001), pp. 10663-10669.

[6] B. Han, P. Serra, A. Amrani, J. Yamanouchi, A. F. M. Marée, L. Edelstein-Keshet, and P. Santamaria, Prevention of diabetes by manipulation of anti-IGRP autoimmunity: high efficiency of a low-affinity peptide., Nat. Med., 11 (2005), pp. 645-652.

[7] D. R. Jackola and H. M. Hallgren, Dynamic phenotypic restructuring of the CD4 and CD8 T-cell subsets with age in healthy humans: a compartmental model analysis., Mech. Ageing Dev., 105 (1998), pp. 241-264.

[8] J. JaCoB AND D. BAltimore, Modelling T-cell memory by genetic marking of memory T cells in vivo., Nature, 399 (1999), pp. 593-597.

[9] C. JANEWAY, Immunobiology : the immune system in health and disease, Garland Science, New York, 2005.

[10] S. M. Lieberman, A. M. Evans, B. Han, T. Takaki, Y. Vinnitskaya, J. A. Caldwell, D. V. Serreze, J. ShaBanowitz, D. F. Hunt, S. G. Nathenson, P. Santamaria, and T. P. DiLorenzo, Identification of the $\beta$ cell antigen targeted by a prevalent population of pathogenic CD8 ${ }^{+} T$ cells in autoimmune diabetes., Proc. Natl. Acad. Sci. U.S.A., 100 (2003), pp. 8384-8388.

[11] R. Maile, B. Wang, W. Schooler, A. Meyer, E. J. Collins, and J. A. Frelinger, Antigen-specific modulation of an immune response by in vivo administration of soluble MHC class I tetramers., J. Immunol., 167 (2001), pp. 3708-3714

[12] A. F. M. Marée, M. Komba, C. Dyck, M. ŁabeçKi, D. T. Finegood, and L. Edelstein-Keshet, Quantifying macrophage defects in type 1 diabetes., J. theor. Biol., 233 (2005), pp. 533-551.

[13] A. F. M. Marée, R. Kublik, D.T Finegood, and L. Edelstein-Keshet, Modelling the onset of type 1 diabetes: can impaired macrophage phagocytosis make the difference between health and disease?, Phil. Trans R. Soc. A., 364 (2006), pp. 1267-1282.

[14] A. F. M. Marée, P. Santamaria, and L. Edelstein-Keshet, Modeling competition among autoreactive CD8 $\left({ }^{+}\right)$ T-cells in autoimmune diabetes: implications for antigen-specific therapy, International Immunology, 18 (2006), pp. 1067-1077.

[15] D. H. MARGulies, Interactions of TCRs with MHC-peptide complexes: a quantitative basis for mechanistic models., Curr. Opin. Immunol., 9 (1997), pp. 390-395.

[16] T. W. McKeithan, Kinetic proofreading in T-cell receptor signal transduction., Proc. Natl. Acad. Sci. U.S.A., 92 (1995), pp. 5042-5046.

[17] D. Moskophidis, F. Lechner, H. Pircher, and R. M. Zinkernagel, Virus persistence in acutely infected immunocompetent mice by exhaustion of antiviral cytotoxic effector T cells., Nature, 362 (1993), pp. 758-761.

[18] B. A. O'Brien, W. E. Fieldus, C. J. Field, And D. T. Finegood, Clearance of apoptotic beta-cells is reduced in neonatal autoimmune diabetes-prone rats., Cell Death. Differ., 9 (2002), pp. 457-464.

[19] B. A. O'Brien, Y. Huang, X. Geng, J. P. Dutz, and D. T. Finegood, Phagocytosis of apoptotic cells by macrophages from NOD mice is reduced., Diabetes, 51 (2002), pp. 2481-2488.

[20] J. T. Opferman, B. T. Ober, and P. G. Ashton-Rickardt, Linear differentiation of cytotoxic effectors into memory T lymphocytes., Science, 283 (1999), pp. 1745-1748.

[21] P. A. Savage, J. J. Boniface, and M. M. Davis, A kinetic basis for T cell receptor repertoire selection during an immune response., Immunity, 10 (1999), pp. 485-492. 
[22] D. F. Tough And J. Sprent, Turnover of naive- and memory-phenotype T cells., J. Exp. Med., 179 (1994), pp. 11271135 .

[23] J. D. Trudeau, C. Kelly-Smith, C. B. Verchere, J. F. Elliott, J. P. Dutz, D. T. Finegood, P. Santamaria, AND R. TAN, Prediction of spontaneous autoimmune diabetes in NOD mice by quantification of autoreactive $T$ cells in peripheral blood., J. Clin. Invest., 111 (2003), pp. 217-223.

[24] S. Valitutti, S. Müller, M. Cella, E. Padovan, and A. Lanzavecchia, Serial triggering of many T-cell receptors by a few peptide-MHC complexes., Nature, 375 (1995), pp. 148-151.

[25] H. Veiga-Fernandes, U. Walter, C. Bourgeois, A. McLean, and B. Rocha, Response of naïve and memory $C D 8^{+} T$ cells to antigen stimulation in vivo., Nat. Immunol., 1 (2000), pp. 47-53.

[26] A. Viola and A. Lanzavecchia, T cell activation determined by $T$ cell receptor number and tunable thresholds., Science, 273 (1996), pp. 104-106.

[27] A. D. Wells, H. Gudmundsdottir, And L. A. Turka, Following the fate of individual T cells throughout activation and clonal expansion: signals from $T$ cell receptor and CD28 differentially regulate the induction and duration of a proliferative response., J. Clin. Invest., 100 (1997), pp. 3173-3183.

\section{Appendix A. Estimation of parameter values.}

We explain our procedure for estimating parameters below, and summarize the values we used in Table B.1.

A.1. Cell turnover rates. The death rate of memory cells is estimated as $\delta_{M}=0.01$ day $^{-1}$ versus $\delta_{E}=0.3$ day $^{-1}$ for effector cells. We here assumed that activated cells have a relatively low death rate, as most are converted into differentiated cells. Consequently, we assumed that $\delta_{A} \approx 0.02$ day $^{-1}$.

A.2. Cell division rates and numbers. We approximated an $8 \mathrm{hr}$ cell cycle for the immune cells, and thereby obtained $\beta \approx 1-6$ day $^{-1}$. The number of memory and effector cells produced per activated $\mathrm{T}$ cell is $0-8$ versus 60 , respectively, according to [14], leading to values for the factors $2^{m_{i}}$.

A.3. Circulating cell levels. According to [14], around 1-10 naive T cells produced by the thymus per day will have the correct specificity. Consequently, $\sigma \approx 1-10$ cells day $^{-1}$. To then determine the competition parameter, $\epsilon$, we first considered the possibility of a QSS for activated T cells of the form $\sigma-\delta_{A} A-\epsilon A^{2}=0$. We found that this cannot be a correct approximation, because the reactivation of memory cells plays a much greater role in sustaining the level of $A$ than the (limited) entry of new naive $\mathrm{T}$ cells from the thymus.

In our subsequent approach, we approximated $M \approx\left(\beta 2^{m_{1}} f_{2} / \delta_{M}\right) A \approx 10^{4}$ circulating memory cells and $E \approx\left(\beta 2^{m_{2}}\left(1-f_{2}\right) / \delta_{E}\right) A \approx 10^{6}$ circulating effector cells. The first of these implies that $\beta f_{2} A \approx 10$ whereas the second that $\beta\left(1-f_{2}\right) A \approx 5 \times 10^{3}$. These approximations lead to $f_{2} \approx 0.002$, and $A \approx$ $1-3 \times 10^{3}$.

Now considering the situation at a high peptide level, near the peak of activated $\mathrm{T}$ cell levels, we have $d A / d t \approx 0$, i.e., $\sigma+\alpha M f_{1}-\left(\beta+\delta_{A}\right) A-\epsilon A^{2} \approx 0$. The relative magnitudes of terms in this equation are as follows: $\sigma$ is very small, and can be neglected in the high-peptide scenario. If $\alpha \approx 1-5$ day $^{-1}$ (which means that on average, a memory cell takes a few hours to be reactivated), and $f_{1} \approx 1$ at high peptide, then $\alpha M f_{1} \approx 1-5 \times 10^{4}$. From above estimates, $\left(\beta+\delta_{A}\right) A \approx \beta A \approx 5 \times 10^{3}$ is of lower order, and $A^{2} \approx 1-4 \times 10^{6}$. The balance is mainly between the terms $\alpha M f_{1}$ and $\epsilon A^{2}$. We can use these figures to estimate the size of the competition parameter, $\epsilon$, from

$$
\epsilon \approx \frac{\alpha M f_{1}}{A^{2}} \approx \frac{1-5 \times 10^{4}}{1-4 \times 10^{6}} \approx 1-5 \times 10^{-2} .
$$

The units of $\epsilon$ are day ${ }^{-1}$ cell $^{-1}$.

A.4. Peptide and beta cell levels. Because peptide level is not directly observed experimentally, its level in the model is on a relative, rather than absolute scale. The important relation is the relative magnitude of $k_{1}, k_{2}$ the parameters that represent the level of peptide at which memory cell production falls off, and T cell activation turns on, respectively. We arbitrarily chose $k_{2}=1$ and $k_{1}=2$. This means that a reasonable scale of peptide level is 0-10 "peptide units". Since peptide timescale is fast, and the peptide variable assumed to be on QSS, only the ratio of the turnover rate $\delta_{p}$ and the production rate, $R$ of the peptide influence the dynamics. Based on the estimated levels of circulating effector $\mathrm{T}$ cells, we used $R \approx 10^{-5}$ per cell per day, and $\delta_{p}=1$ per day to give QSS value of peptide in the range of 0-10. We also use a relative scale for the level of remaining beta cells: i.e., $B$ represents the fraction of beta cells still remaining, so $0 \leq B \leq 1$.

The removal of peptide by macrophages, by diffusion, and other influences is assumed to be in the range of $\delta_{p} \approx 1$ day $^{-1}$. When effector cell levels are high, $E \approx 10^{6}$ cells, leads to the approximation $p \approx 10 \approx R B E / \delta_{4} \approx R \times 10^{6}$. This leads to an estimate $R \approx 10^{-5}$ peptide units day ${ }^{-1}$ cell $^{-1}$. 
A.5. Typical values of variables. The results of above ball-park estimates lead to the following ranges of the variables concerned:

$$
A \approx 1-2 \times 10^{3}, \quad M \approx 1-5 \times 10^{4}, \quad E \approx 1-6 \times 10^{6}, \quad p \approx 1-10, \quad B \approx 1 .
$$

The populations of the three types of $\mathrm{T}$ cells differ by many orders of magnitude. We therefore scaled each variable in terms of some power of ten for convenient graphics. The scaling considerations are discussed in the next section.

\section{Appendix B. Scaling the equations.}

Let $A=A^{*} \bar{A}, M=M^{*} \bar{M}, E=E^{*} \bar{E}$, etc, where stars denote numerical values and overbars denote quantities carrying units. We keep time in units of days, i.e., time is not scaled. Then Equations (3.1)(3.7) can be rewritten as follows:

$$
\begin{aligned}
\frac{d A^{*}}{d t} & =\left(\frac{\sigma}{\bar{A}}+\left(\frac{\alpha \bar{M}}{\bar{A}}\right) M^{*}\right) f_{1}(p)-\left(\beta+\delta_{A}\right) A^{*}-(\epsilon \bar{A})\left(A^{*}\right)^{2}, \\
\frac{d M^{*}}{d t} & =\left(\beta 2^{m_{1}} \frac{\bar{A}}{\bar{M}}\right) f_{2}(p) A^{*}-f_{1}(p) \alpha M^{*}-\delta_{M} M^{*}, \\
\frac{d E^{*}}{d t} & =\left(\beta 2^{m_{2}} \frac{\bar{A}}{\bar{E}}\right)\left(1-f_{2}(p)\right) A^{*}-\delta_{E} E^{*}, \\
\frac{d B^{*}}{d t} & =-(\kappa \bar{E}) E^{*} B^{*} \\
\text { QSS : } \quad p & =\left(\frac{R \bar{E} \bar{B}}{\delta_{p}}\right) E^{*} B^{*},
\end{aligned}
$$

Since peptide is already in arbitrary units, we did not rescale the peptide or the functions $f_{1}, f_{2}$. Dropping the ${ }^{*}$ 's we thus obtained a new system of (scaled) equations

$$
\begin{aligned}
\frac{d A}{d t} & =\left(a_{6}+a_{7} M\right) f_{1}(p)-a_{8} A-a_{9} A^{2}, \\
\frac{d M}{d t} & =a_{10} f_{2}(p) A-f_{1}(p) a_{7} a_{16} M-a_{11} M, \\
\frac{d E}{d t} & =a_{12}\left(1-f_{2}(p)\right) A-a_{13} E, \\
\frac{d B}{d t} & =-a_{17} E B, \\
\text { QSS : } \quad p & =\left(a_{14} / a_{15}\right) E B,
\end{aligned}
$$

where the new parameters so defined are as follows:

$$
\begin{gathered}
a_{6}=\frac{\sigma}{\bar{A}}, \quad a_{7}=\frac{\alpha \bar{M}}{\bar{A}}, \quad a_{8}=\beta+\delta_{A}, \quad a_{9}=\epsilon \bar{A} \\
a_{10}=\beta 2^{m_{1}} \frac{\bar{A}}{\bar{M}}, \quad a_{11}=\delta_{M}, \quad a_{16}=\frac{\bar{A}}{\bar{M}} \\
a_{12}=\beta 2^{m_{2}} \frac{\bar{A}}{\bar{E}}, \quad a_{13}=\delta_{E}, \quad a_{17}=\kappa \bar{E}, \quad a_{14}=R \bar{E} \bar{B}, \quad a_{15}=\delta_{p}
\end{gathered}
$$

The original variables, $A, M, E$ differ by 6 orders of magnitude. We therefore selected a scaling of the main variables in various powers of ten, so as to display all results on a common coordinate system within the range of 1-10 units. To do so, we scaled variables by selecting the following reference scales:

$$
\bar{A}=10^{3}, \quad \bar{M}=10^{4}, \quad \bar{E}=10^{6}, \quad \bar{p}=10, \quad \bar{B}=1 .
$$




\begin{tabular}{|c|c|c|c|c|}
\hline par. & meaning & default value & units & ref. \\
\hline$\sigma$ & influx naive $\mathrm{T}$ cells from thymus & $1-10$ & cell day $^{-1}$ & {$[3,14]$} \\
\hline$\alpha$ & rate of production of $\mathrm{A}$ per $\mathrm{M}$ & $1-5$ & $\mathrm{day}^{-1}$ & estimated \\
\hline$\beta$ & rate of cell division & $1-6$ & $\mathrm{day}^{-1}$ & typical \\
\hline$\delta_{A}$ & death rate, activated $\mathrm{T}$ cells & $\approx 0.01$ & day $^{-1}$ & {$[22,7]$} \\
\hline$\delta_{M}$ & death rate, memory $\mathrm{T}$ cells & $\approx 0.01$ & day $^{-1}$ & {$[22,7,14]$} \\
\hline$\delta_{E}$ & death rate, effector $\mathrm{T}$ cells & 0.3 & day $^{-1}$ & {$[5,14]$} \\
\hline$\overline{\delta_{p}}$ & peptide turnover rate & $0-1$ & day $^{-1}$ & estimated \\
\hline$\epsilon$ & T cell competition parameter & $1-5 \times 10^{-2}$ & $(\text { cell day })^{-1}$ & estimated \\
\hline$k_{1}$ & peptide level for $\frac{1}{2} \max$ activation & 2 & peptide units & arbitrary \\
\hline$k_{2}$ & peptide level for $\frac{1}{2}$ max memory cells & 1 & peptide units & arbitrary \\
\hline$m$ & Hill coeff. for memory cell production & 2 & - & {$[14]$} \\
\hline$n$ & Hill coeff. for $\mathrm{T}$ cell activation & 3 & - & {$[14]$} \\
\hline $2^{m_{1}}$ & $\begin{array}{l}\text { maximum number of memory cells pro- } \\
\text { duced per proliferating } \mathrm{T} \text { cell }\end{array}$ & 8 & - & {$[27,20,25]$} \\
\hline $2^{m_{2}}$ & $\begin{array}{l}\text { number of effector cells produced per pro- } \\
\text { liferating } \mathrm{T} \text { cell }\end{array}$ & 60 & - & {$[20,25]$} \\
\hline$a$ & $\begin{array}{l}\text { maximal fraction of memory cells pro- } \\
\text { duced }\end{array}$ & $<1$ & - & fitted \\
\hline$R$ & peptide accumulation rate & $10^{-5}$ & day $^{-1}$ cell $^{-1}$ & estimated \\
\hline$\kappa$ & beta cell killing per effector $\mathrm{T}$ cell & $0.14 \times 10^{-6}$ & day $^{-1}$ cell $^{-1}$ & fitted \\
\hline
\end{tabular}

TABLE B.1

Default "NOD mouse" parameter values used to simulate the model. See Appendix A for a description of how these values were estimated.

\begin{tabular}{|c|c|c|c|c|}
\hline $\begin{array}{c}\text { Scaled } \\
\text { parameter }\end{array}$ & $\begin{array}{c}\text { original } \\
\text { parameter }\end{array}$ & $\begin{array}{c}\text { default } \\
\text { value }\end{array}$ & $\begin{array}{c}\text { increase } \\
+10 \%\end{array}$ & $\begin{array}{c}\text { decrease } \\
-10 \%\end{array}$ \\
\hline$a_{1}$ & $n$ & 2 & $\mathrm{~S}, \rightarrow$ & $10 \mathrm{P}, \leftarrow$ \\
\hline$a_{2}$ & $k_{1}$ & 2 & $\mathrm{~S}, \rightarrow$ & $10 \mathrm{P}, \leftarrow$ \\
\hline$a_{3}$ & $m$ & 3 & $\mathrm{~S}, \rightarrow$ & $9 \mathrm{P}, \leftarrow$ \\
\hline$a_{4}$ & $a$ & 0.7 & $\mathrm{NC}, 3 \mathrm{P}$ & $\mathrm{S}, \rightarrow$ \\
\hline$a_{5}$ & $k_{2}$ & 1 & $9 \mathrm{P} \leftarrow$ & $\mathrm{S}, \rightarrow$ \\
\hline$a_{6}$ & $\sigma$ & 0.02 & $4 \mathrm{P} \leftarrow$ & $\mathrm{S}, \rightarrow$ \\
\hline$a_{7}$ & $\alpha$ & 20 & $7 \mathrm{P} \leftarrow$ & $\mathrm{S}, \rightarrow$ \\
\hline$a_{8}$ & $\beta+\delta_{A}$ & 1 & $\mathrm{~S} \rightarrow$ & $9 \mathrm{P}, \leftarrow$ \\
\hline$a_{9}$ & $\epsilon$ & 1 & $\mathrm{NC}, 3 \mathrm{P}$ & $\mathrm{NC}, 3 \mathrm{P}$ \\
\hline$a_{10}$ & $\beta 2^{m}$ & 1 & $8 \mathrm{P} \leftarrow$ & $\mathrm{S}, \rightarrow$ \\
\hline$a_{11}$ & $\delta_{M}$ & 0.01 & $\mathrm{NC}, 3 \mathrm{P}$ & $\mathrm{NC}, 3 \mathrm{P}$ \\
\hline$a_{12}$ & $\beta 2^{m}$ & 0.1 & $4 \mathrm{P} \leftarrow$ & $\mathrm{S}, \rightarrow$ \\
\hline$a_{13}$ & $\delta_{E}$ & 0.3 & $\mathrm{~S} \rightarrow$ & $7 \mathrm{P}, \leftarrow$ \\
\hline$a_{14}$ & $R$ & 50 & $4 \mathrm{P} \leftarrow$ & $\mathrm{S}, \rightarrow$ \\
\hline$a_{15}$ & $\delta_{p}$ & 1 & $\mathrm{~S}, \rightarrow$ & $4 \mathrm{P}, \leftarrow$ \\
\hline$a_{16}$ & $\mathrm{Scale}$ & 0.1 & $\mathrm{~S}, \rightarrow$ & $5 \mathrm{P}, \leftarrow$ \\
\hline$a_{17}$ & $\kappa$ & 0.14 & $1 \mathrm{P}, \rightarrow$ & $4 \mathrm{P}, \leftarrow$ \\
\hline
\end{tabular}

Parameter sensitivity. The default value of each (scaled) parameter is shown, and the effect of a $10 \%$ increase and decrease recorded. (See Appendix $C$ for the definition of scaled parameters.) Original parameter values produce a stable limit cycle (i.e., dynamics between the Hopf and the homoclinic bifurcations). $\rightarrow$ denotes a shift towards the homoclinic bifurcation, $\leftarrow$ denotes a shift towards the Hopf bifurcation, or even beyond it, to the stable steady state. S denotes return to healthy state, $P$ signifies how many peaks (cycles) are are seen before the homoclinic bifurcation occurs. NC means little or no change. 
Appendix C. XPP code. A typical XPP file used to produce figures in this paper is given below.

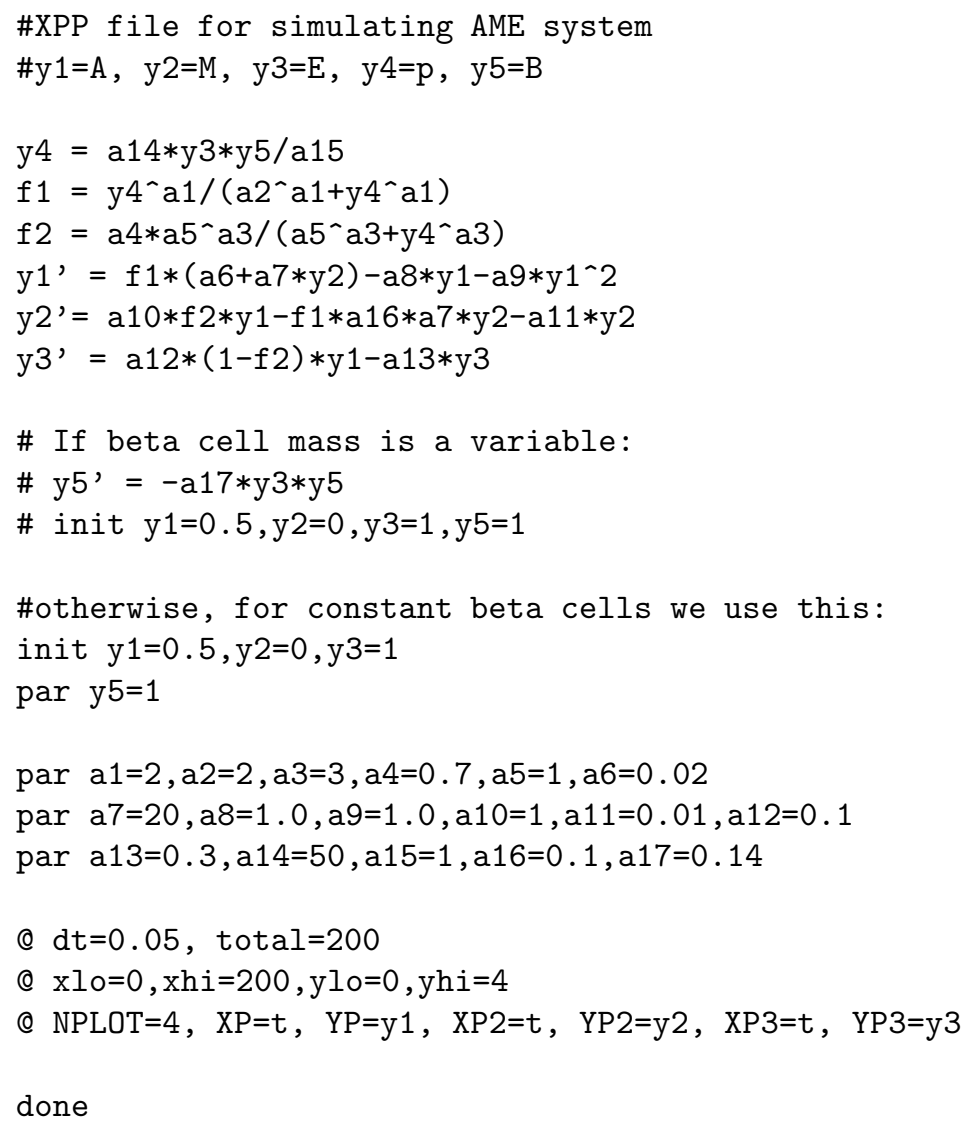

Appendix D. List of abbreviations.

We used the following abbreviations.

APC: antigen presenting cell

CTL: cytotoxic T lymphocyte

DC: dendritic cell

IGRP: islet-specific glucose 6 phosphatase catalytic subunit related protein

MHC: Major Histocompatibility Complex

NOD: non-obese diabetic mouse

ODE: ordinary differential equation model

T1D: type 1 diabetes

TCR: T cell receptor

QSS: quasi-steady state 